\title{
Surface composition and taxonomic classification of a group of near-Earth and Mars-crossing asteroids
}

\author{
Juan A. Sanchez ${ }^{\mathrm{a}, *}$, René Michelsen ${ }^{1}$, Vishnu Reddy ${ }^{\mathrm{b}, 2}$, Andreas Nathues $^{\mathrm{c}}$ \\ ${ }^{a}$ Institut für Planetologie, Westfälische Wilhelms-Universität Münster, Germany \\ ${ }^{b}$ Department of Space Studies, University of North Dakota, Grand Forks, ND 58202, USA \\ ${ }^{c}$ Max Planck Institut für Sonnensystemforschung, Max Planck Str.2, 37191 Katlenburg-Lindau, Germany
}

\begin{abstract}
In the past, constraining the surface composition of near-Earth asteroids (NEAs) has been difficult due to the lack of high quality near-IR spectral data $(0.7-2.5 \mu \mathrm{m})$ that contain mineralogically diagnostic absorption bands. Here we present visible $(\sim 0.43-0.95 \mu \mathrm{m})$ and nearinfrared $(\sim 0.7-2.5 \mu \mathrm{m})$ spectra of nine NEAs and five Mars-crossing asteroids (MCs). The studied NEAs are: 4055 Magellan, 19764 (2000 NF5), 89830 (2002 CE), 138404 (2000 HA24), 143381 (2003 BC21), 159609 (2002 AQ3), 164121 (2003 YT1), 241662 (2000 KO44) and 2007 ML13. The studied MCs are: 1656 Suomi, 2577 Litva, 5407 (1992 AX), 22449 Ottijeff and 47035 (1998 WS). The observations were conducted with the NTT at La Silla, Chile, the $2.2 \mathrm{~m}$ telescope at Calar Alto, Spain, and the IRTF on Mauna Kea, Hawai'i. The taxonomic classification (Bus system) of asteroids showed that all observed MC asteroids belong to the S-complex, including the S, Sr and Sl classes. Seven of the NEAs belong to the S-complex, including the $\mathrm{S}$, Sa, Sk and Sl classes, and two NEAs were classified as V-types. The classification of the NEA 164121 (2003 YT1) as a V-type was made on the basis of its near-infrared spectrum since no visible spectrum is available for this asteroid. A mineralogical analysis was performed on six of the asteroids (those for which near-IR spectra were obtained or previously available). We found that three asteroids (241662 (2000 KO44), 19764 (2000 NF5), 138404 (2000 HA24)) have mafic silicate compositions consistent with ordinary chondrites, while three others (4055 Magellan, 164121 (2003 YT1), 5407 (1992 AX)) are pyroxene-dominated basaltic achondrite assemblages. In the case of 5407 (1992 AX) we found that its basaltic surface composition contrasts its taxonomic classification as a S-type.
\end{abstract}

Keywords:

Asteroids, Near-Earth objects, Spectroscopy, Infrared observations, Meteorites

\section{Introduction}

Among the small body population, near-Earth asteroids (NEAs) are defined as asteroids having perihelion distances of $\leq 1.3 \mathrm{AU}$. The NEA population shows a great diversity in terms of taxonomic classification, including almost all classes of asteroids found in the main belt

\footnotetext{
${ }^{*}$ Corresponding author at: Max Planck Institut für Sonnensystemforschung, Max Planck Str.2, 37191 Katlenburg-Lindau, Germany.

Email address: sanchez@mps.mpg.de (Juan A. Sanchez)

${ }^{1}$ Visiting Scientist at the Max Planck Institut für Sonnensystemforschung.

${ }^{2}$ Visiting Astronomer at the Infrared Telescope Facility, which is operated by the University of Hawaii under Cooperative Agreement No. NNX-08AE38A with the National Aeronautics and Space Administration, Science Mission Directorate, Planetary Astronomy Program.
} 
(Binzel et al., 2002). However, of all taxonomic types, S-complex asteroids are the dominant class among NEAs. This high proportion of S-complex is caused in part by a selection effect, due to the fact that S-complex asteroids have higher albedos than C-types (the dominant class among all main-belt asteroids), making them easier to discover. In addition, there are some regions in the main belt that contribute more than others to the delivery of objects into the inner solar system. According to some dynamical models (e.g., Morbidelli and Nesvorny, 1999; Bottke et al., 2000, 2002; Morbidelli et al., 2002), the 3:1 mean-motion resonance with Jupiter (occurring at $\sim 2.5 \mathrm{AU}$ ), and the $\nu_{6}$ secular resonance at the inner asteroid belt (where Scomplex are most common) provide the most significant fraction of objects to the near-Earth population.

Based on their orbital parameters NEAs are divided into three subgroups called Amor, Apollo, and Aten asteroids (Shoemaker et al., 1979). Some of these objects are also classified as potentially hazardous asteroids (PHAs), which are defined as objects whose minimal orbital intersection distance (MOID) with Earth is $\leq 0.05 \mathrm{AU}$. In addition to this dynamical classification, there is another group of objects called Mars-crossing asteroids (MCs), whose orbits cross or approach that of Mars, and are characterized by perihelion distances $1.30<$ $q<1.66$ AU. Similarly to NEAs, the MC population is dominated by S-complex asteroids (Angeli and Lazzaro, 2002; Binzel et al., 2004). With time, MCs can eventually become NEAs as a result of close approaches with Mars, that move them into a resonance, and from there, to the near-Earth space (Michel et al., 2000).

NEAs are of great interest for study, and due to their proximity they could be the source of some of the meteorites found on Earth. Thus, establishing links between the spectral properties of NEAs and those measured in the laboratory for meteorite samples is fundamental to determine their possible parent bodies (Burbine et al., 2002; Binzel et al., 2002). Recent studies show that about $2 / 3$ of the observed NEAs have surface compositions consistent with LL chondrites, which represent only about $10 \%$ of the ordinary chondrites falls (Vernazza et al., 2008; de León et al., 2010; Dunn et al., 2013). These results contradict the general assumption that the spectral characteristics of the most common type of NEAs should be consistent with those of the most common meteorites to fall on Earth. The reason for this discrepancy still remains unclear.

NEAs are also interesting for study because they are relatively easy to reach compared to their counterparts in the main belt. Some NEAs have low velocities with respect to Earth $(\Delta \mathrm{V})$, which make them especially accessible for sample-return and manned missions (e.g., Sears et al., 2000; Abell et al., 2009; Reddy et al., 2012a). Furthermore, it is now recognized the threat that some NEAs could represent for civilization in the eventuality of collision (e.g., Chapman and Morrison, 1994; Morrison et al., 2002). Hence, having a better understanding of their physical properties could help us to develop mitigation strategies in the future.

In this work we report visible (VIS) and near-infrared (NIR) spectroscopic observations of five MCs and nine NEAs. Although limited, our sample is diverse and comprises both basaltic an ordinary chondrite-like asteroids. Furthermore, three objects are classified as PHAs and one object is considered as a possible target for robotic and manned missions. Thus, the present work seeks to obtain new compositional information that could help tracing the origin of some meteorites, contribute in planning future robotic and manned missions, and contribute in the development of mitigation strategies. In addition, one of the goals of this paper is to study the compositional trend in the MC population and compare it with the NEA population. This will help us understand the source regions from which the NEAs are derived, and if the same compositional types dominate both populations. This is a preliminary study since our current sample is small. The selection of these particular asteroids for study was influenced by different factors that limit the number of objects that can be observed for a certain period of time. Magnitude limitations imposed by the telescope size, the zenith distance of the asteroids at the 
time of the observations (i.e., in order to minimize distortion from atmospheric extinction the asteroid must be observed at rather small airmass $<1.5$ ), and the proximity of the targets to the galactic plane, are among the factors that normally reduce an initial list of several tens of targets to only a few of them. In Sect. 2 we describe the observations and data reduction procedure. In Sect. 3 we present the results, including the taxonomic classification and mineralogical analysis of the asteroids. In Sect. 4, we present and summarize our conclusions.

\section{Observations and data reduction}

\subsection{Visible spectra}

The VIS spectroscopic observations were conducted with the New Technology Telescope (NTT) at La Silla, Chile on August 2010, and with the $2.2 \mathrm{~m}$ telescope at Calar Alto, Spain, on October 2010.

The VIS spectra obtained with the NTT were acquired using the EFOSC2 spectrograph, equipped with a $2048 \times 2048$ pixels Loral/Lesser CCD (detector \# 40), with a pixel size of 15 $\mu \mathrm{m}$, corresponding to 0 ".12/pixel. The disperser element used was the Grism \#1 with a 5" slit width, providing an effective wavelength range of $\sim 0.43-0.95 \mu \mathrm{m}$. The spectrograph used with the $2.2 \mathrm{~m}$ telescope was CAFOS. Due to a technical problem two different detectors were used during the observing run at Calar Alto. The first two nights (October 05 and 06) the Lor\#11i $2 \mathrm{k} \times 2 \mathrm{k}$ chip (pixel size of $15 \mu \mathrm{m}$ ) was used. During the last night (October 07), data were acquired with the SITe- $1 \mathrm{~d} 2 \mathrm{k} \times 2 \mathrm{k}$ chip $(24 \mu \mathrm{m}$ pixel, i.e., 0".53/pixel). This is the standard CCD for CAFOS. The disperser element was the Grism R-400 with a 5" slit width. The covered wavelength range is $\sim 0.5-0.92 \mu \mathrm{m}$.

Asteroid spectra were acquired using a nodding technique in which the object is alternated between two different slit positions (A and B) following the sequence ABBA. During the observations the slit was oriented along the parallactic angle in order to minimize the effects of differential atmospheric refraction. To obtain the relative reflectance, solar analog stars were observed at similar air masses as the asteroids. For each night, flat fields, bias and arc line spectra were acquired. Observational circumstances for the studied objects are presented in Table 1.

The NTT is capable of differential tracking, however previous studies found that for exposure times longer than $\sim 10$ minutes, NEAs tend to move out of the slit (Michelsen et al., 2006). Therefore, exposures were limited to $600 \mathrm{~s}$. In the case of the $2.2 \mathrm{~m}$ telescope, due to its excellent tracking capability the exposure time for the asteroids was $900 \mathrm{~s}$.

The data reduction was performed using ESO-MIDAS following the same procedure described in (e.g., Michelsen et al., 2006; Nathues, 2010). The steps involved in the reduction process are: (1) sky background removal by subtracting A from B and B from A, (2) flat-field correction, (3) median filtering for removal of cosmic hits, (4) extraction of the one-dimensional spectra, (5) wavelength calibration using Helium and Argon arc spectra (for the NTT data) and $\mathrm{HgHeRb}$ arc spectra (for the Calar Alto data), (6) extinction correction (for the NTT data), using La Silla's mean extinction curve (Tüg, 1977), (7) co-adding of individual spectra to increase the $\mathrm{S} / \mathrm{N},(8)$ division of the asteroids spectrum by the spectrum of a solar analog star, and (9) normalization of the spectra to unity at $0.55 \mu \mathrm{m}$.

After reducing the data we noticed that the spectra obtained with the $2.2 \mathrm{~m}$ telescope and the Lor\#11i CCD exhibit strong fringing longwards of $0.7 \mu \mathrm{m}$. This can be seen in Fig. 1 that shows the spectrum of asteroid (1656) Suomi obtained with the two detectors used during the observations, the Lor\#11i (top panel) and the SITe-1d (bottom panel). Since the fringing pattern could not be removed during the reduction process, in the present work we only report the data obtained with the SITe-1d CCD. 


\subsection{Near-infrared spectra}

The NIR observations were conducted remotely with the NASA Infrared Telescope Facility (IRTF) on Mauna Kea, Hawai'i, on January 2007, October 2009 and July 2010. NIR spectra were obtained with the SpeX instrument (Rayner et al., 2003), equipped with a Raytheon Aladdin $31024 \times 1024 \mathrm{InSb}$ array. Spectral data were acquired using Spex in its low resolution $(\mathrm{R} \sim 150)$ prism mode with a 0.8 " slit width. The covered wavelength range is $\sim 0.7-2.5 \mu \mathrm{m}$. The procedure to acquire NIR spectra is essentially the same to that followed to acquire VIS spectra. The main difference is that in addition to the solar analogs, and in order to correct for telluric water vapor features, local standard stars are also observed. Due to a higher and more variable background at NIR wavelengths exposures were limited to $120 \mathrm{~s}$. SpeX data were reduced with Spextool (Cushing et al., 2004). NIR spectra are normalized to unity at $1.4 \mu \mathrm{m}$. Observational circumstances for the studied asteroids are presented in Table 1.

Table 1: Observational circumstances. The columns in this table are: object number and designation, orbit, UT date, telescope and instrument used, number of exposures, phase angle $(\alpha)$, V-magnitude at the time of observation, air mass and solar analog used. Asteroids with the $\left(^{*}\right)$ symbol have a MOID $\leq$ $0.05 \mathrm{AU}$ and therefore are also classified as PHAs. The integration time with NTT/EFOSC2 was $600 \mathrm{~s}$, with CA $2.2 \mathrm{~m} / \mathrm{CAFOS}$ was $900 \mathrm{~s}$, and with IRTF/SpeX was $120 \mathrm{~s}$.

\begin{tabular}{|c|c|c|c|c|c|c|c|c|}
\hline Object & Orbit & UT date & $\begin{array}{l}\text { Telescope and } \\
\text { instrument }\end{array}$ & Exp & $\alpha\left(^{\circ}\right)$ & mag. (V) & Air mass & Solar analog \\
\hline 1656 Suomi & $\mathrm{MC}$ & 07-Oct-2010 & CA $2.2 \mathrm{~m} / \mathrm{CAFOS}$ & 4 & 10.4 & 14.5 & 1.52 & 16 Cyg B \\
\hline 2577 Litva & $\mathrm{MC}$ & 17-Aug-2010 & NTT/EFOSC2 & 3 & 12.5 & 15.8 & 1.36 & Land 115-271 \\
\hline $5407(1992 \mathrm{AX})$ & $\mathrm{MC}$ & 12-Aug-2010 & NTT/EFOSC2 & 4 & 22.8 & 16.6 & 1.06 & Land $112-1333$ \\
\hline 5407 (1992 AX) & $\mathrm{MC}$ & 07-Jan-2007 & IRTF/SpeX & 20 & 16.2 & 15.8 & 1.25 & Hyades 64 \\
\hline 22449 Ottijeff & $\mathrm{MC}$ & 12-Aug-2010 & NTT/EFOSC2 & 4 & 10.5 & 17.1 & 1.24 & Land 112-1333 \\
\hline 47035 (1998 WS) & $\mathrm{MC}$ & 13-Aug-2010 & NTT/EFOSC2 & 6 & 8.7 & 17.7 & 1.22 & HD 1835 \\
\hline 4055 Magellan & Amor & 12-Aug-2010 & NTT/EFOSC2 & 2 & 52.8 & 15.7 & 1.49 & Land 112-1333 \\
\hline 4055 Magellan & Amor & 21-July-2010 & IRTF/SpeX & 10 & 49.4 & 15.9 & 1.02 & 16 Cyg B \\
\hline 19764 (2000 NF5) & Amor & 12-Aug-2010 & NTT/EFOSC2 & 3 & 36.8 & 16.5 & 1.38 & Land $112-1333$ \\
\hline $89830(2002 \mathrm{CE})$ & $\operatorname{Amor}^{(*)}$ & 07-Oct-2010 & $\mathrm{CA} 2.2 \mathrm{~m} / \mathrm{CAFOS}$ & 2 & 68.1 & 16.3 & 1.41 & Hyades 64 \\
\hline 138404 (2000 HA24) & $\operatorname{Apollo}^{(*)}$ & 17-Aug-2010 & NTT/EFOSC2 & 5 & 2.7 & 16.9 & 1.08 & Land $112-1333$ \\
\hline $143381(2003 \mathrm{BC} 21)$ & Amor & 17-Aug-2010 & NTT/EFOSC2 & 4 & 12.7 & 17.2 & 1.51 & Land $115-271$ \\
\hline 143381 (2003 BC21) & Amor & $07-O c t-2010$ & CA $2.2 \mathrm{~m} / \mathrm{CAFOS}$ & 4 & 27.4 & 16.6 & 1.20 & Hyades 64 \\
\hline 159609 (2002 AQ3) & Amor & 16-Aug-2010 & NTT/EFOSC2 & 4 & 38.8 & 16.7 & 1.25 & Land $112-1333$ \\
\hline 164121 (2003 YT1) & $\operatorname{Apollo}^{(*)}$ & 20-Oct-2009 & IRTF/SpeX & 20 & 70.4 & 14.8 & 1.24 & Hyades 64 \\
\hline $241662(2000 \mathrm{KO} 44)$ & Amor & 13-Aug-2010 & NTT/EFOSC2 & 2 & 54.7 & 17.1 & 1.35 & HD 1835 \\
\hline 2007 ML13 & Amor & 17-Aug-2010 & NTT/EFOSC2 & 6 & 14.7 & 18.0 & 1.12 & Land 112-1333 \\
\hline
\end{tabular}

\section{Results}

\subsection{Taxonomic classification}

The taxonomic classification of the asteroids was made following the system developed by Bus (1999), which is comprised of 26 classes that include three major complexes (S-, C- and Xcomplex). The classification was obtained using a computer program that includes the steps described in Bus (1999). Asteroid VIS spectra are shown in Figs. 2 and 3. The taxonomic classification of the observed asteroids is presented in Table 2. If the asteroid was previously classified the taxonomic class and reference are indicated.

Five of the observed objects are MC asteroids, and according to our analysis all belong to the S-complex (see Tables 1 and 2). These objects have been observed and classified by previous studies (Bus and Binzel, 2002a, b; ; Lazzaro et al., 2004; Binzel et al., 2004). Discrepancies 


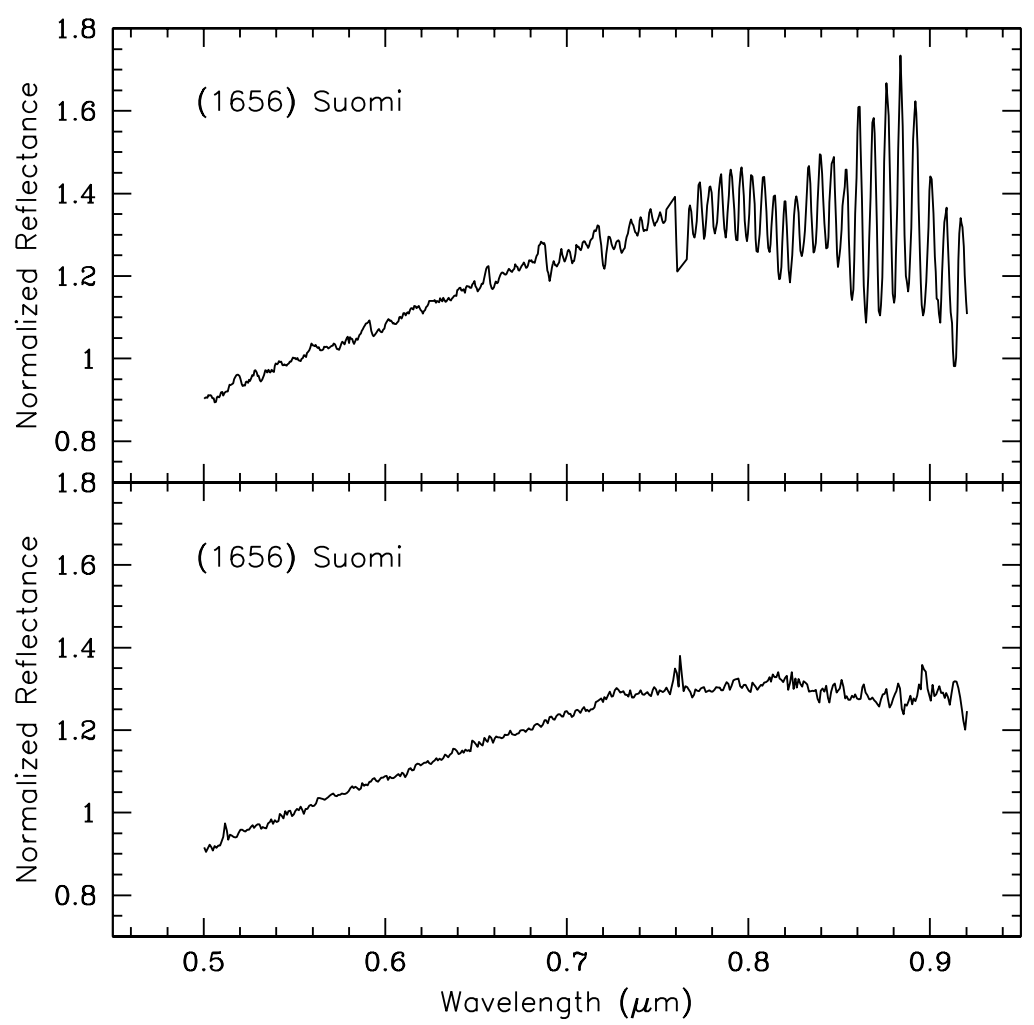

Figure 1: The spectrum of asteroid (1656) Suomi obtained with the $2.2 \mathrm{~m}$ telescope using two different detectors, the Lor\#11i (top panel) and the SITe-1d (bottom panel). The spectrum obtained with the Lor\#11i CCD exhibits strong fringing longwards of $0.7 \mu \mathrm{m}$. In the present work we only report the data obtained with the SITe-1d CCD.

between the taxonomic classification assigned by us and that by previous work were found for two of the asteroids (1656 Suomi and 5407). According to our results asteroid (1656) Suomi belongs to the S-complex, however due to the scattering in the data beyond $0.8 \mu \mathrm{m}$ we were not able to establish its subtype within the S-complex. Lazzaro et al. (2004) classified this asteroid as Ld. In the case of asteroid 5407 it was classified by us as S-type, while Bus and Binzel (2002a b) classified it as Sk (same complex, different subtype). Figure 4 shows the spectra of asteroid (1656) Suomi and 5407 compared with those obtained by Lazzaro et al. (2004) and Bus and Binzel (2002a, b) , respectively. As noted by Bus et al. (2002), these kind of discrepancies are often reported when the spectra of asteroids observed by different surveys are compared. These differences could be attributed to several factors like instrumental effects and viewing geometry effects (Bus et al., 2002). The latter can manifest themselves as phase reddening, which produces an increase of the spectral slope and variations in the intensity of the absorption bands (Sanchez et al., 2012). The poor weather conditions during our observing run at Calar Alto could also contribute to explain the difference between the taxonomic classification assigned by us and that by previous studies.

Of the nine NEAs observed, only two, 4055 Magellan and 164121 (2003 YT1), don't belong to the S-complex. The VIS spectrum of 4055 Magellan exhibits a very deep 1- $\mu$ m absorption band characteristic of pyroxene assemblages (e.g., Cloutis and Gaffey, 1991; Gaffey et al., 2002; Moskovitz et al., 2010). Our classification of this asteroid as a V-type is consistent with previous studies (Lazzaro et al., 2004; Binzel et al., 2004). For 164121 (2003 YT1) we only have 
Table 2: Taxonomic classification (Bus) of the observed asteroids. The measured spectral slope for each asteroid is given. If the asteroid has been classified by previous work the reference is indicated by (a) (Bus and Binzel, 2002a, b) , (b) (Lazzaro et al., 2004) and (c) (Binzel et al., 2004). Asteroid 143381 (2003 BC21) was observed with two telescopes, the $\left(^{*}\right)$ symbol was assigned to the spectrum obtained with the $2.2 \mathrm{~m}$ telescope at Calar Alto. In the case of asteroid 164121 (2003 YT1) there is no VIS spectrum, therefore it is not possible to classify it in the Bus system. However, based on its NIR spectral characteristics we concluded that this NEA is a V-type.

\begin{tabular}{|c|c|c|c|}
\hline Object & Slope $\left(\mu \mathrm{m}^{-1}\right)$ & Tax (previous work) & Tax (present work) \\
\hline 1656 Suomi & 1.080 & $\mathrm{Ld}^{(b)}$ & $\mathrm{S}-$ complex \\
2577 Litva & 0.819 & $\mathrm{Sl}^{(b)}$ & $\mathrm{Sl}$ \\
$5407(1992 \mathrm{AX})$ & 0.575 & $\mathrm{Sk}^{(a)}$ & $\mathrm{S}$ \\
22449 Ottijeff & 0.567 & $\mathrm{~S}^{(a)}$ & $\mathrm{S}$ \\
47035 (1998 WS) & 0.462 & $\mathrm{Sr}^{(a)}$ & $\mathrm{Sr}$ \\
4055 Magellan & -0.153 & $\mathrm{~V}^{(b, c)}$ & $\mathrm{V}$ \\
$19764(2000 \mathrm{NF} 5)$ & 0.441 & - & $\mathrm{S}$ \\
$89830(2002 \mathrm{CE})$ & 0.382 & - & $\mathrm{S}$ \\
$138404(2000 \mathrm{HA} 24)$ & 0.449 & - & $\mathrm{S}$ \\
$143381(2003 \mathrm{BC} 21)$ & 0.528 & - & $\mathrm{S}$ \\
$143381(2003 \mathrm{BC} 21)^{(*)}$ & 0.354 & - & $\mathrm{Sk}$ \\
$159609(2002 \mathrm{AQ} 3)$ & 0.419 & - & $\mathrm{S}$ \\
$164121(2003 \mathrm{YT} 1)$ & - & - & $\mathrm{Sl}$ \\
$241662(2000 \mathrm{KO} 44)$ & 1.066 & - & \\
$2007 \mathrm{ML} 13$ & 0.797 & & \\
\hline
\end{tabular}

a NIR spectrum, therefore we couldn't classify it under the Bus system. However, based on its mineralogical analysis (see below) we found that this NEA is a V-type asteroid as well (see Figure 5). NEAs 19764 (2000 NF5), 138404 (2000 HA24), 143381 (2003 BC21) and 89830 (2002 CE) were classified as S-types, while 241662 (2000 KO44), 159609 (2002 AQ3) and 2007 ML13 were classified as Sa, Sk and Sl, respectively. 

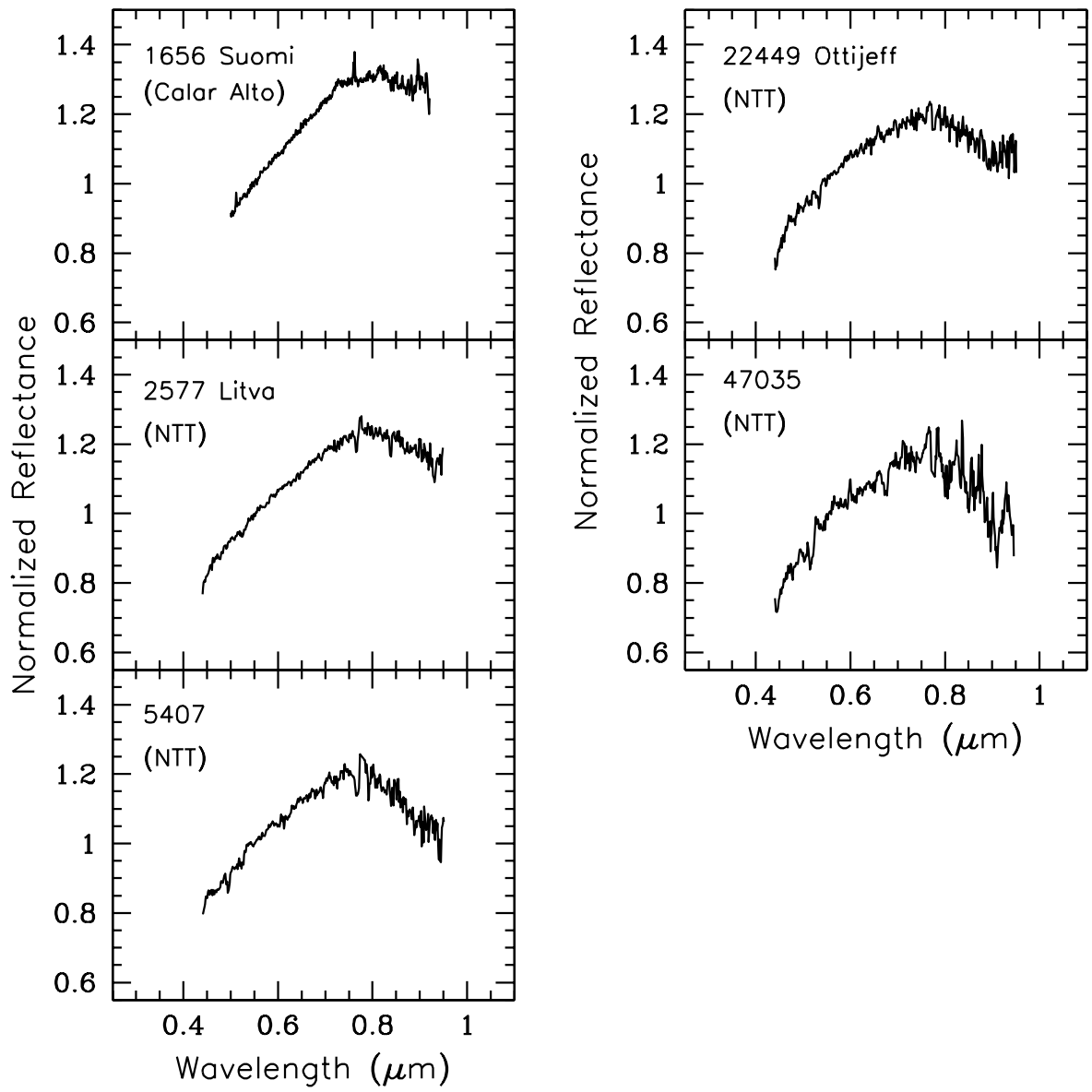

Figure 2: Spectra of MC asteroids obtained with the NTT and the $2.2 \mathrm{~m}$ telescope. The numerical designation for each asteroid is given. All spectra are normalized to unity at $0.55 \mu \mathrm{m}$. 

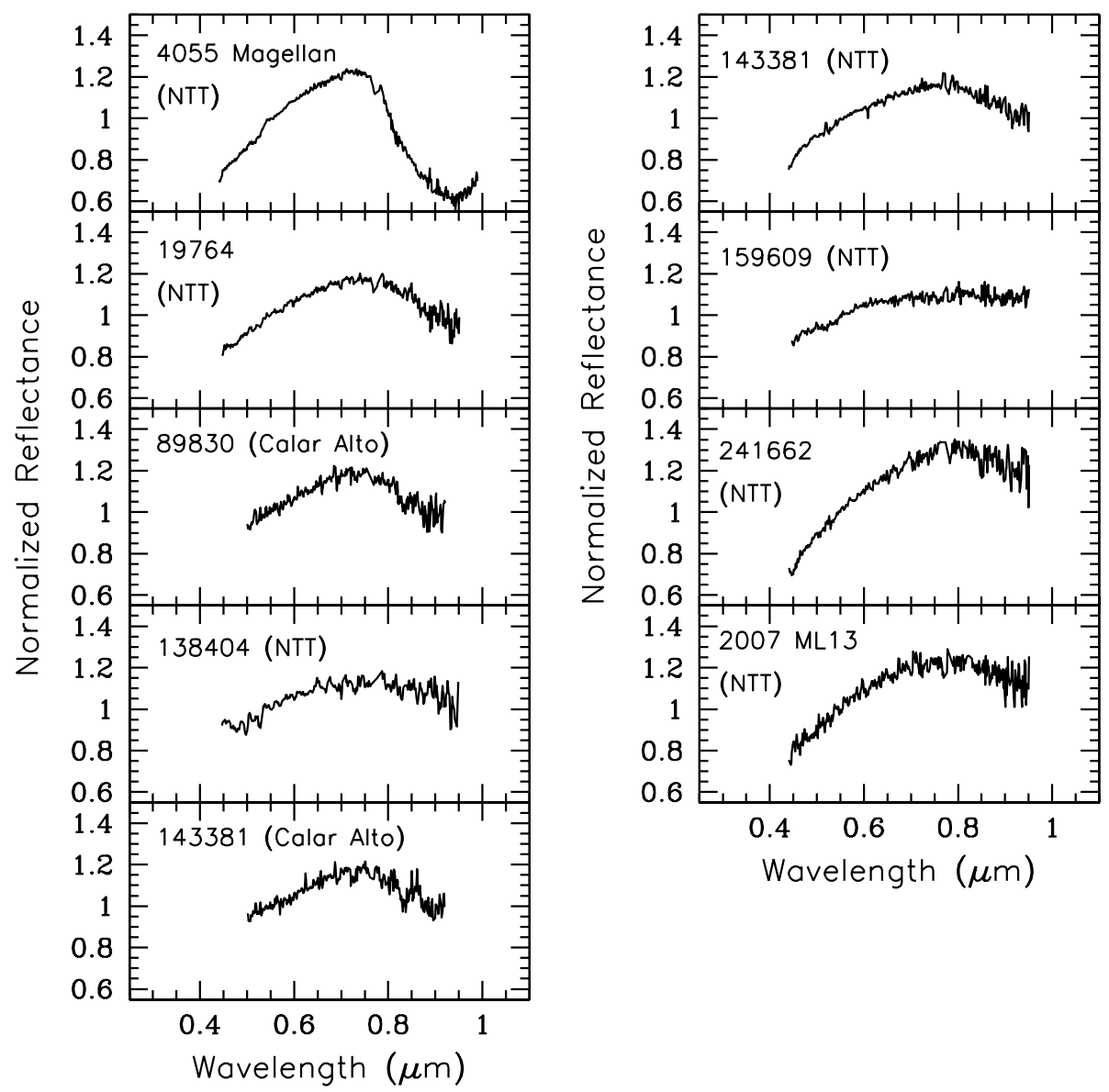

Figure 3: Spectra of NEAs obtained with the NTT and the $2.2 \mathrm{~m}$ telescope. The numerical designation for each asteroid is given. All spectra are normalized to unity at $0.55 \mu \mathrm{m}$. Asteroid 143381 (2003 BC21) was observed with both telescopes, due to bad weather conditions the spectrum obtained with the 2.2 $\mathrm{m}$ telescope at Calar Alto looks noisier than the one obtained with the NTT. 


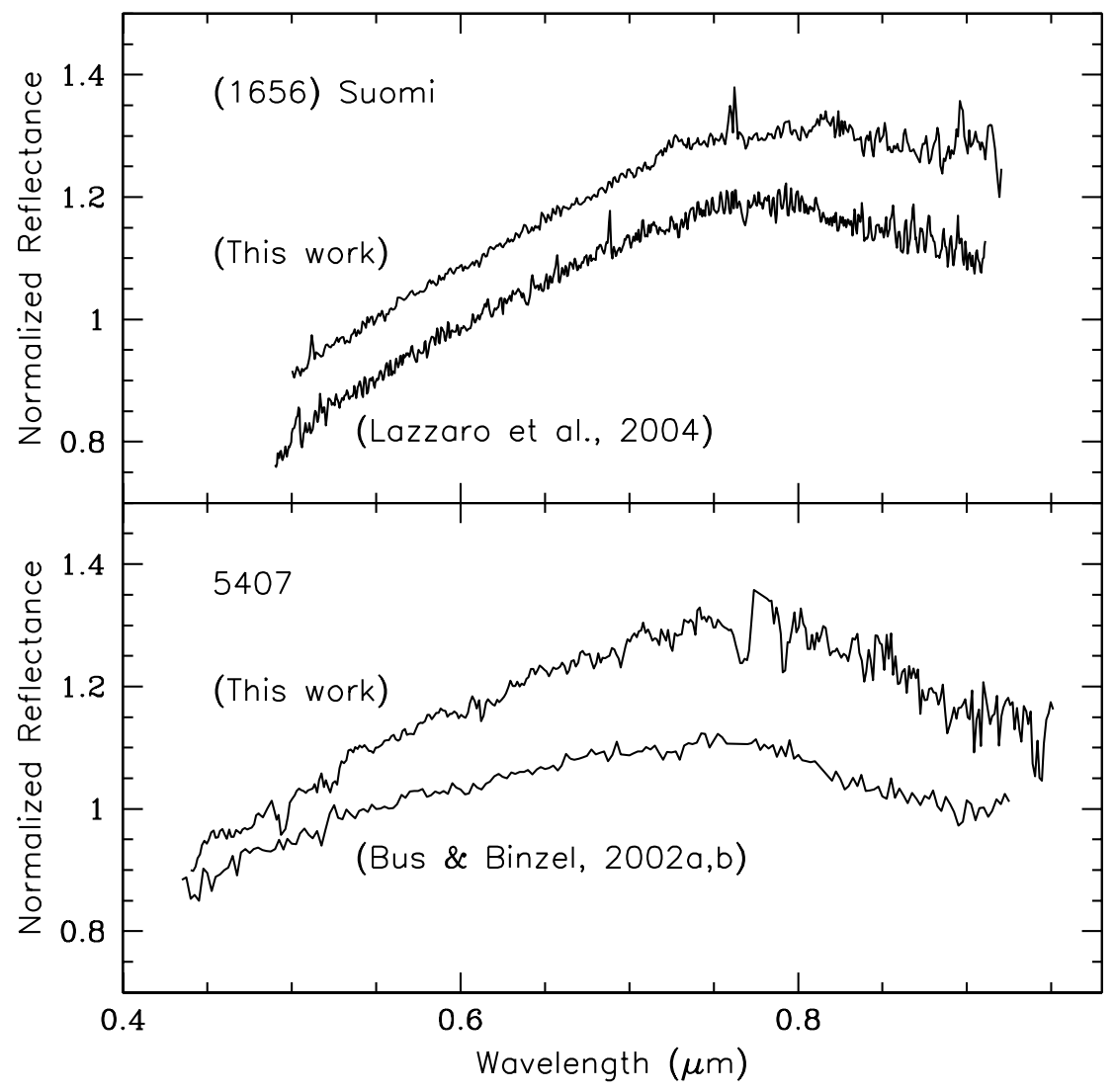

Figure 4: Spectra of asteroid (1656) Suomi (top panel) and 5407 (bottom panel) compared with those obtained by Lazzaro et al. (2004) and Bus and Binzel (2002a,b), respectively. Spectra are normalized to unity at $0.55 \mu \mathrm{m}$, and are offset for clarity. 


\subsection{Mineralogical analysis}

Our observations with the IRTF include NIR spectra of asteroids 4055 Magellan, 164121 (2003 YT1), and 5407 (1992 AX). For three of the NEAs that we obtained visible spectra, 241662 (2000 KO44), 19764 (2000 NF5) and 138404 (2000 HA24), there is also available NIR spectra $(0.8-2.5 \mu \mathrm{m})$ from the MIT-UH-IRTF Joint Campaign for NEO Spectral Reconnaissance (NEOSR). These spectra were acquired with the IRTF and SpeX. All the data (with the exception of 164121 (2003 YT1)) were combined with the VIS spectra to increase the wavelength coverage, allowing the mineralogical characterization of these asteroids. For that purpose, spectral band parameters, band centers and Band Area Ratios (BAR) for each VIS-NIR spectrum were measured in the same way as in Sanchez et al. (2012). In the case of NEA 164121 (2003 YT1), the wavelength range covered $(\sim 0.7-2.5 \mu \mathrm{m})$ was sufficient to allow us to measure these band parameters.

The VIS-NIR spectra of asteroids 4055 Magellan and 5407 (1992 AX), and the NIR spectrum of 164121 (2003 YT1) are shown in Fig. 5. The spectra of these objects show absorption bands characteristics of pyroxene assemblages, one centered near 0.9-1 $\mu \mathrm{m}$ (Band I) and the other at $\sim 1.9-2 \mu \mathrm{m}$ (Band II). These absorption bands are caused by the presence of $\mathrm{Fe}^{2+}$ cations located in the M2 crystallographic site (Adams, 1974, 1975; Burns, 1993). The VIS-NIR spectra of asteroids 241662 (2000 KO44), 19764 (2000 NF5) and 138404 (2000 HA24) (shown in Fig. 6) exhibit the two absorption bands characteristics of olivine-orthopyroxene assemblages.

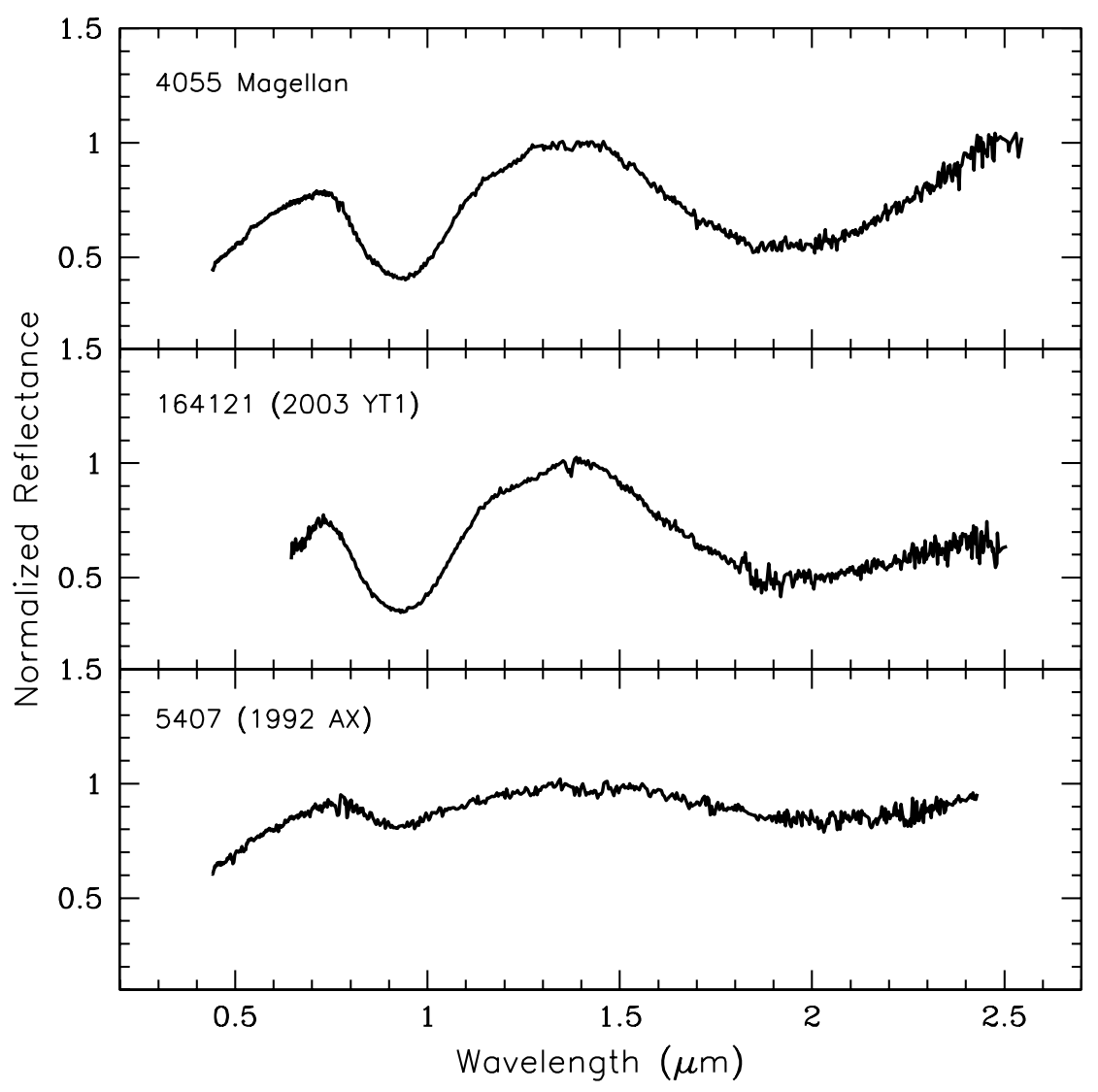

Figure 5: VIS-NIR spectra of asteroids 4055 Magellan (top panel) and 5407 (1992 AX) (bottom panel), and NIR spectrum of 164121 (2003 YT1) (middle panel). All spectra are normalized to unity at $1.4 \mu \mathrm{m}$. 


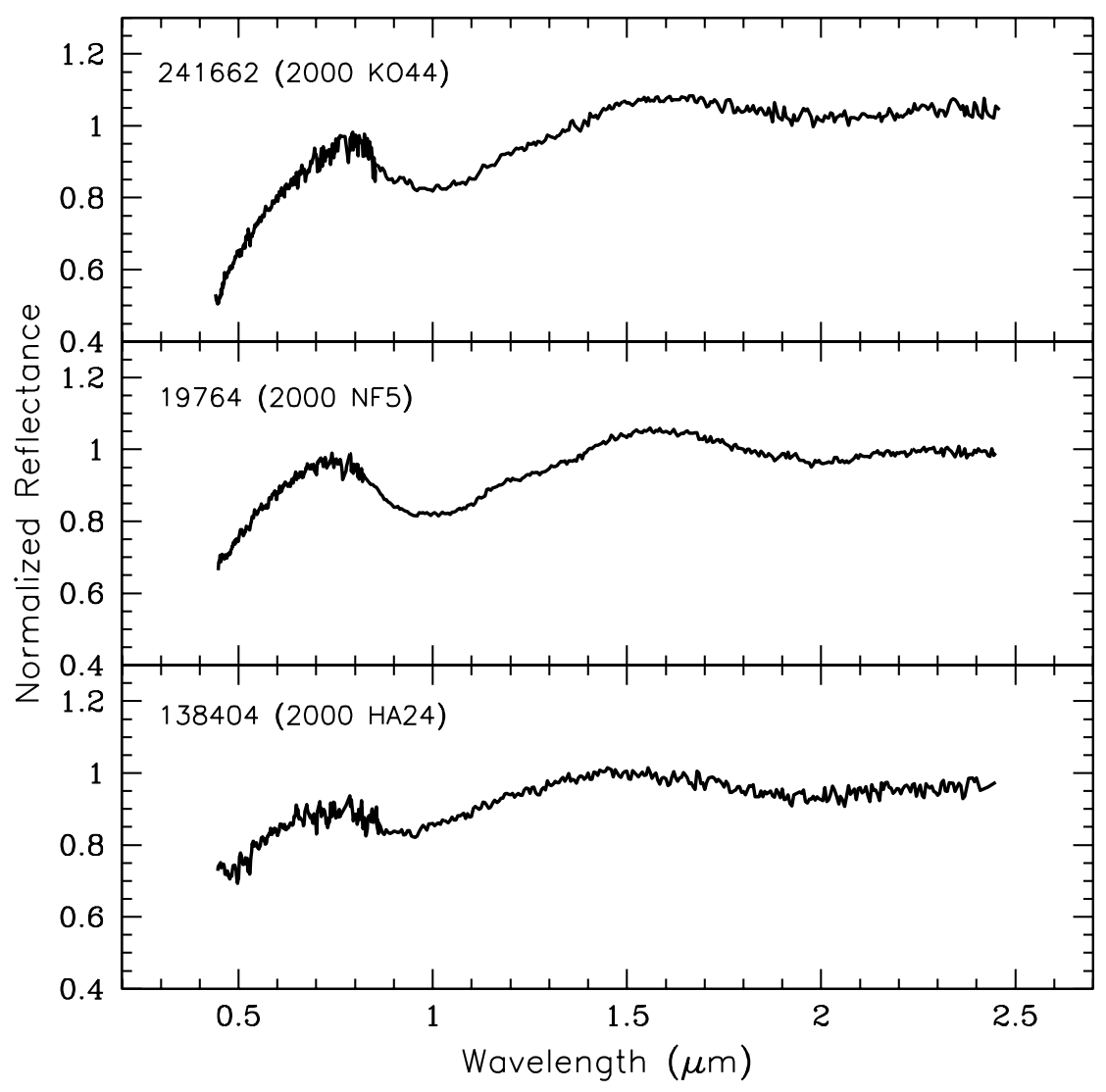

Figure 6: VIS-NIR spectra of asteroids 241662 (2000 KO44) (top panel), 19764 (2000 NF5) (middle panel) and 138404 (2000 HA24) (bottom panel). NIR asteroid spectra were obtained from the NEOSR survey (http://smass.mit.edu/minus.html). All spectra are normalized to unity at $1.4 \mu \mathrm{m}$.

As stated earlier, the VIS-NIR spectra of 4055 Magellan and 5407 (1992 AX), and the NIR spectrum of 164121 (2003 YT1) show spectral characteristics of basaltic asteroids. Therefore, to the band parameters, we applied the temperature corrections derived by Reddy et al. (2012c). In addition, the pyroxene compositions of these objects were determined using the equations derived by Burbine et al. (2007). With these equations we can determine the molar contents of ferrosilite (Fs) and wollastonite (Wo) for basaltic asteroids.

For asteroids 241662 (2000 KO44), 19764 (2000 NF5) and 138404 (2000 HA24), which exhibit spectral characteristics of olivine-orthopyroxene assemblages, temperature corrections to band parameters were applied using the equations obtained by Sanchez et al. (2012). The mafic silicate compositions of these objects were determined using the equations derived by Dunn et al. (2010). With these equations it is possible to determine the molar contents of fayalite $(\mathrm{Fa})$ in olivine and ferrosilite $(\mathrm{Fs})$ in pyroxene in olivine-orthopyroxene assemblages. In addition, the mineral abundances $(o l /(o l+p x))$ were calculated from the BAR values using the relationship found by Dunn et al. (2010). This relationship was obtained from the analysis of ordinary chondrites and therefore can be applied to asteroids with similar mineralogies. Spectral band parameters and mineral chemistry values for the studied asteroids are presented in Table 3.

Apart from the mineralogical analysis, the spectral band parameters (Band I center and 
BAR) were also used to classify these asteroids in the system introduced by Gaffey et al. (1993), which divides the S-population into seven main compositional subgroups designated S(I)-S(VII). Fig. 7 shows the measured Band I center versus BAR for 4055 Magellan (open square), 5407 (1992 AX) (open circle) and 164121 (2003 YT1) (open triangle). These three asteroids are located in the rectangular zone (BA), which includes the pyroxene-dominated basaltic achondrite assemblages (Gaffev et al., 1993). Within the uncertainties, asteroids 241662 (2000 KO44), 19764 (2000 NF5) and 138404 (2000 HA24) are classified as S(IV) under the system of Gaffey et al. (1993). In Fig. 7 are also depicted the measured Band I center versus BAR for 241662 (2000 KO44) (filled triangle), 19764 (2000 NF5) (filled circle) and 138404 (2000 HA24) (filled square).

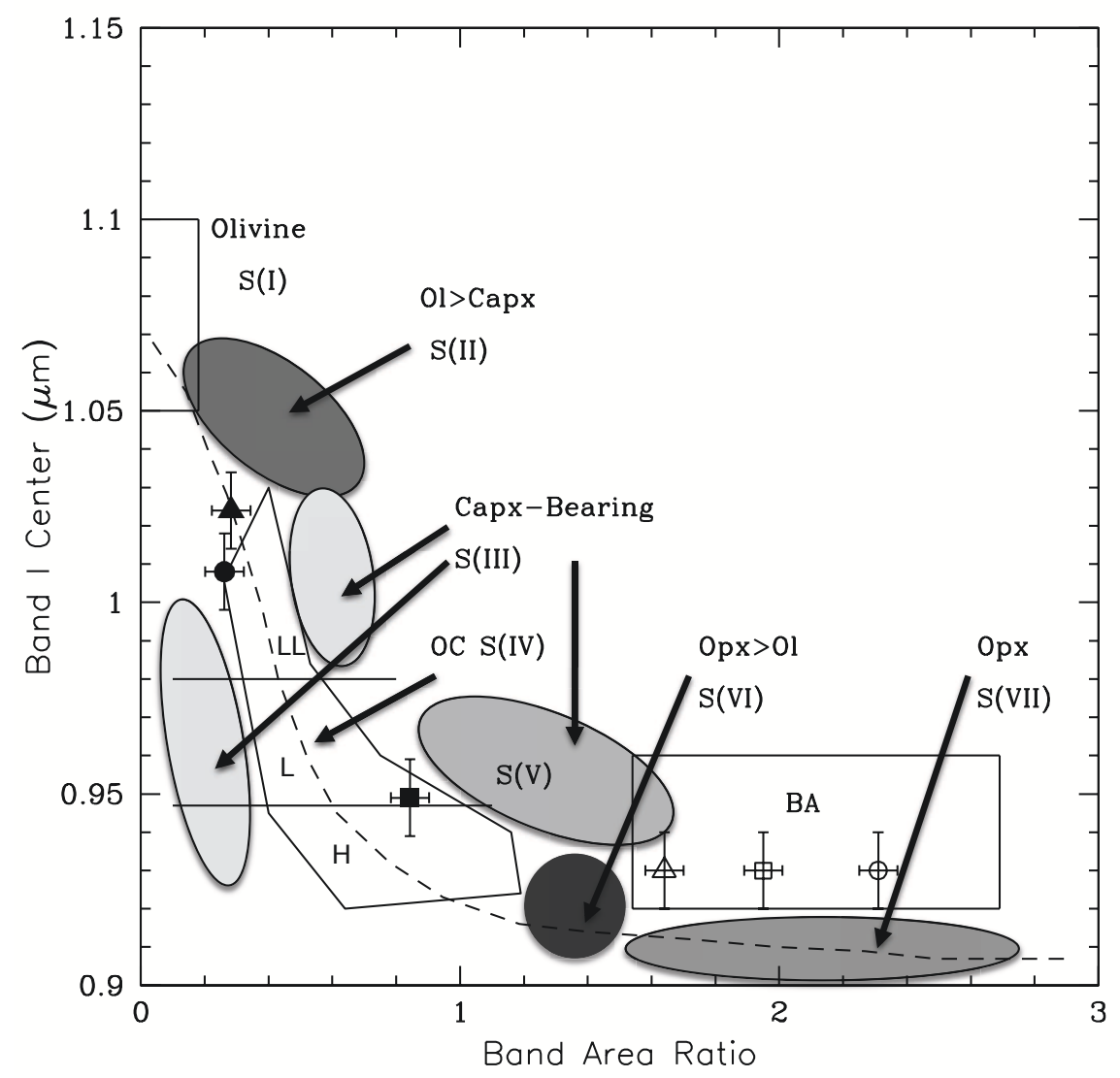

Figure 7: Plot of the Band I center versus BAR for 241662 (2000 KO44) (filled triangle), 19764 (2000 NF5) (filled circle), 138404 (2000 HA24) (filled square), 4055 Magellan (open square), 5407 (1992 AX) (open circle), and 164121 (2003 YT1) (open triangle). The average 1- $\sigma$ error bars were calculated as in Sanchez et al. (2012). The rectangular zone (BA) includes the pyroxene-dominated basaltic achondrite assemblages (Gaffey et al., 1993). The polygonal region, corresponding to the S(IV) subgroup, represents the mafic silicate components of ordinary chondrites (OC). The dashed curve indicates the location of the olivine-orthopyroxene mixing line (Cloutis et al., 1986). The horizontal lines represent the approximate boundaries for ordinary chondrites found by Dunn et al. (2010). 
For NEA 4055 Magellan, the Band I center is located at $0.93 \pm 0.01 \mu \mathrm{m}$ and the Band II center is at $1.92 \pm 0.02 \mu \mathrm{m}$ with a BAR of $1.95 \pm 0.06$. After plotting the band centers of this asteroid on the Band I vs. Band II center plot (Fig. 8, filled circle), we found that this object has a surface assemblage dominated by orthopyroxene. For comparison we also plotted the band centers of asteroid (4) Vesta (star symbol) measured by Reddy et al. (2012c). The spectrum of this asteroid shows an inflection at $\sim 1.3 \mu \mathrm{m}$, which is visible in Type A clinopyroxene, olivineorthopyroxene mixtures and plagioclase (Burns, 1993). From our calculation we determined that the pyroxene chemistry of 4055 Magellan is $\mathrm{Fs}_{35} \mathrm{En}_{58} \mathrm{Wo}_{7}$. These values are consistent with those measured for howardites $\left(\mathrm{Fs}_{24-42}, \mathrm{Wo}_{2-8}\right)$, and cumulate eucrites $\left(\mathrm{Fs}_{30-44}, \mathrm{Wo}_{6-10}\right)$ (Mittlefehldt et al., 1998). Our results are in agreement with those found by Burbine et al. (2009), which gave a pyroxene chemistry of $\mathrm{Fs}_{36} \mathrm{En}_{57} \mathrm{Wo}_{7}$ for Magellan. In Fig. 9 we plot the molar content of Wo versus Fs for 4055 Magellan, depicted as a filled circle. The approximated range of pyroxene chemistries found for howardites, eucrites and diogenites are shown as dashedline boxes.

For the PHA 164121 (2003 YT1), the Band I and Band II centers are located at $0.93 \pm 0.01 \mu \mathrm{m}$ and $1.90 \pm 0.02 \mu \mathrm{m}$, respectively. The calculated BAR is 1.64 \pm 0.06 . Similarly to 4055 Magellan, this asteroid exhibits a surface assemblage dominated by orthopyroxene (filled triangle in Fig. 8). Like Magellan, the spectrum of 164121 (2003 YT1) shows an inflection at $\sim 1.3 \mu \mathrm{m}$. For this NEA the pyroxene chemistry was determined to be $\mathrm{Fs}_{32} \mathrm{En}_{62} \mathrm{Wo}_{6}$. These values are consistent with the measured ranges for both howardites and cumulate eucrites, and are similar to the values reported by Abell et al. (2004), who estimated a pyroxene chemistry of $\mathrm{Fs}_{32} \mathrm{En}_{60} \mathrm{Wo}_{8}$ for this asteroid. The molar contents of Wo and Fs for 164121 (2003 YT1) are shown in Fig. 9 (filled triangle).

The measured Band I and Band II centers of the MC 5407 (1992 AX) are located at $0.93 \pm 0.01 \mu \mathrm{m}$ and $2.01 \pm 0.02 \mu \mathrm{m}$, respectively, with a BAR of $2.31 \pm 0.06$. The position of the band centers, which are represented in Fig. 8 as a filled square, indicates that this asteroid has a surface assemblage possibly dominated by clinopyroxenes. As in the previous cases, the pyroxene chemistry of the asteroid was calculated, given the value of $\mathrm{Fs}_{45} \mathrm{En}_{45} \mathrm{Wo}_{10}$. This pyroxene chemistry is consistent with the range estimated for non-cumulate eucrites $\left(\mathrm{Fs}_{43-55}\right.$, Wo9-15) (Mittlefehldt et al., 1998). The molar contents of Wo and Fs calculated for 5407 (1992 AX) are indicated in Fig. 9 with a filled square. The surface composition of this asteroid contrast with its taxonomic classification derived from the VIS spectrum, which classify it as a S-type. However, the analysis of NIR spectra of individual S-type asteroids has proved that this class can include both differentiated and primitive objects (Gaffey et al., 1993). Asteroid 5407 represents one of those cases in which a S-type exhibits signs of differentiation. This object is also a good example of the limitations of taxonomic classification, and shows how the assumption that objects belonging to the same class share similar mineralogies can lead to a misinterpretation about the real nature of the asteroid.

For NEA 241662 (2000 KO44), the Band I center is located at $1.02 \pm 0.01 \mu \mathrm{m}$ and the Band II center is at $1.99 \pm 0.02 \mu \mathrm{m}$ with a BAR of $0.28 \pm 0.06$. The Band I center and BAR values of this object place it in the upper limit of the polygonal region defined by Gaffey et al. (1993), and just on the olivine-orthopyroxene mixing line of Cloutis et al. (1986) (Fig. 7). Within the error bars this asteroid is classified as a S(IV) in the system of Gaffey et al. (1993). Using the equations derived by Dunn et al. (2010) we determined that the olivine and pyroxene chemistries are $\mathrm{Fa}_{31}\left(\mathrm{Fo}_{69}\right)$ and $\mathrm{Fs}_{25}$, respectively. These values are consistent with those derived for LL ordinary chondrites by Dunn et al. (2010), which give a range of $\mathrm{Fa}_{(25-33)}$ for fayalite and $\mathrm{Fs}_{(21-27)}$ for ferrosilite. The molar contents of Fa vs. Fs for 241662 (2000 KO44) are shown in Fig. 10 as a filled triangle. For comparison we also plotted the values for LL (open triangles), $\mathrm{L}$ (open circles) and H (x symbols) ordinary chondrites from Nakamura et al. (2011). From 


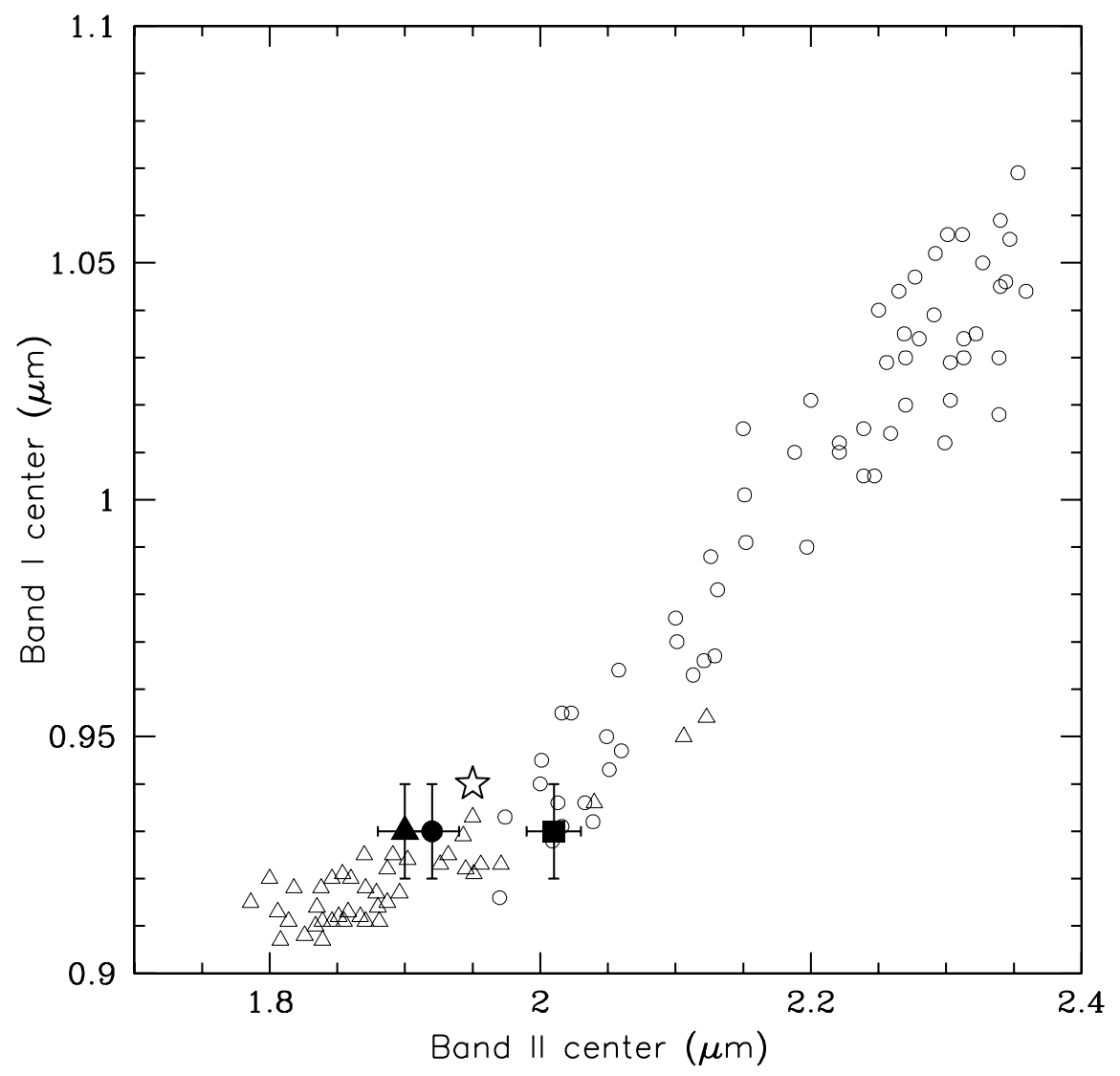

Figure 8: Plot of Band I center vs. Band II center for 4055 Magellan (filled circle), 164121 (2003 YT1) (filled triangle), and 5407 (1992 AX) (filled square). The average 1- $\sigma$ error bars were calculated as in Sanchez et al. (2012). Open triangles represent measured band centers for orthopyroxenes from Adams (1974), open circles correspond to measured band centers for clinopyroxenes from Cloutis and Gaffey 1991). For comparison we plotted the band centers of (4) Vesta (star symbol) measured by Reddy et al. (2012c). The error bars are smaller than the symbol.

the BAR value we estimated that the olivine and pyroxene percentages for this asteroid are 0.66 and 0.34 , respectively. These values are also in agreement with X-ray diffraction (XRD) measurements reported by Dunn et al. (2010) for LL ordinary chondrites, that give $o l /(o l+p x)$ values in the range of $0.58-0.69$.

The Band I center of NEA 19764 (2000 NF5) is located at 1.01 $\pm 0.01 \mu \mathrm{m}$ and the Band II center is at $1.99 \pm 0.02 \mu \mathrm{m}$ with a BAR of $0.26 \pm 0.06$. The Band II center of this asteroid is consistent with those of low calcium $(\sim 1.8-2.1 \mu \mathrm{m})$ and high calcium pyroxenes $(\sim 1.97-2.35$ $\mu \mathrm{m}$ ). The band parameters of 19764 (2000 NF5) are slightly offset to the left of the olivineorthopyroxene mixing line (see Fig. 7). This shift could be due to the presence of small amounts of high calcium pyroxene along with olivine and orthopyroxene in the assemblage. Within the error bars, 19764 (2000 NF5) belongs to the S(IV) compositional subgroup. For this asteroid the calculated $\mathrm{Fa}$ and $\mathrm{Fs}$ content $\left(\mathrm{Fa}_{30}\right.$ and $\left.\mathrm{Fs}_{25}\right)$, as well as the $o l /(o l+p x)$ ratio of 0.66 , fall into the range of LL ordinary chondrites. Fig. 10 shows the molar contents of Fa and Fs for 19764 (2000 NF5) (filled circle).

In the case of the PHA 138404 (2000 HA24), the Band I and Band II centers are located at $0.95 \pm 0.01 \mu \mathrm{m}$ and $1.98 \pm 0.02 \mu \mathrm{m}$, respectively, with a BAR of $0.84 \pm 0.06$. This asteroid plots 


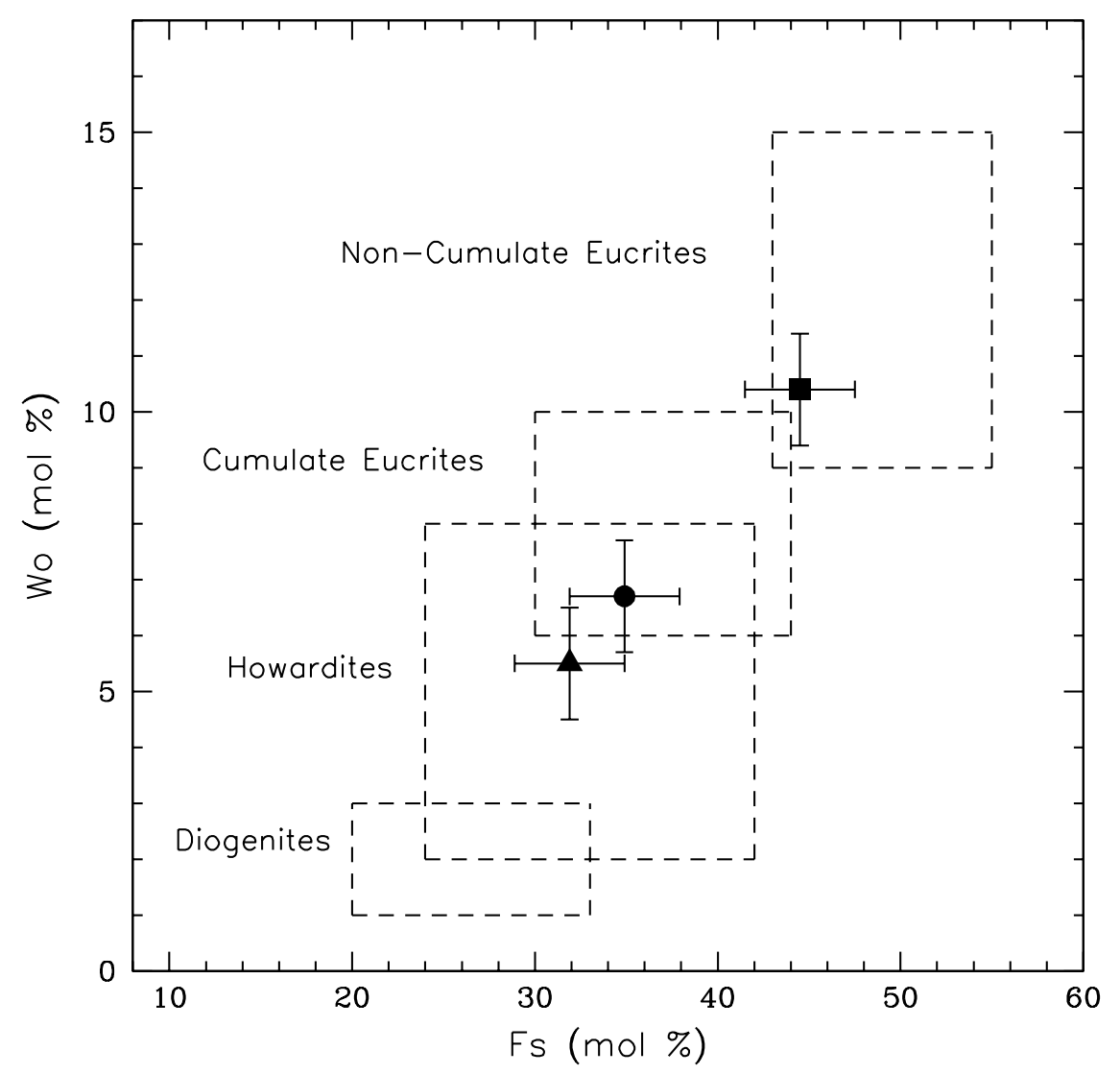

Figure 9: Molar contents of Wo vs. Fs for NEAs 4055 Magellan (filled circle), 164121 (2003 YT1) (filled triangle), and 5407 (1992 AX) (filled square). The error bars correspond to the values determined by Burbine et al. (2007), $3 \mathrm{~mol} \%$ for $\mathrm{Fs}$ and $1 \mathrm{~mol} \%$ for Wo. The approximated range of pyroxene chemistries for howardites, non-cumulate eucrites, cumulate eucrites, and diogenites from Mittlefehldt et al. (1998) are indicated as dashed-line boxes.

in the $\mathrm{S}(\mathrm{IV})$ region (Fig. 7) at the boundary between $\mathrm{H}$ and $\mathrm{L}$ chondrites. As can be seen in Fig. 7 the band parameters of this object are offset to the right of the olivine-orthopyroxene mixing line, which is an indication for the presence of high calcium pyroxene on the surface of the asteroid (Gaffey et al., 1993). According to our calculations, the olivine and pyroxene chemistries for this asteroid are $\mathrm{Fa}_{22}\left(\mathrm{~F}_{78}\right)$ and $\mathrm{Fs}_{18}$, respectively. These values are in the borderline of those derived by Dunn et al. (2010) for L (Fa $21-27$ and $\left.\mathrm{Fs}_{17-23}\right)$ and $\mathrm{H}\left(\mathrm{Fa}_{15-21}\right.$ and $\left.\mathrm{Fs}_{13-19}\right)$ ordinary chondrites. The $o l /(o l+p x)$ ratio of 0.52 calculated for 138404 (2000 HA24) falls into the range of $\mathrm{H}$ ordinary chondrites (0.46-0.60) measured by Dunn et al. (2010). The derived olivine and pyroxene chemistries of 138404 (2000 HA24) are shown in Fig. 10 as a filled square. 


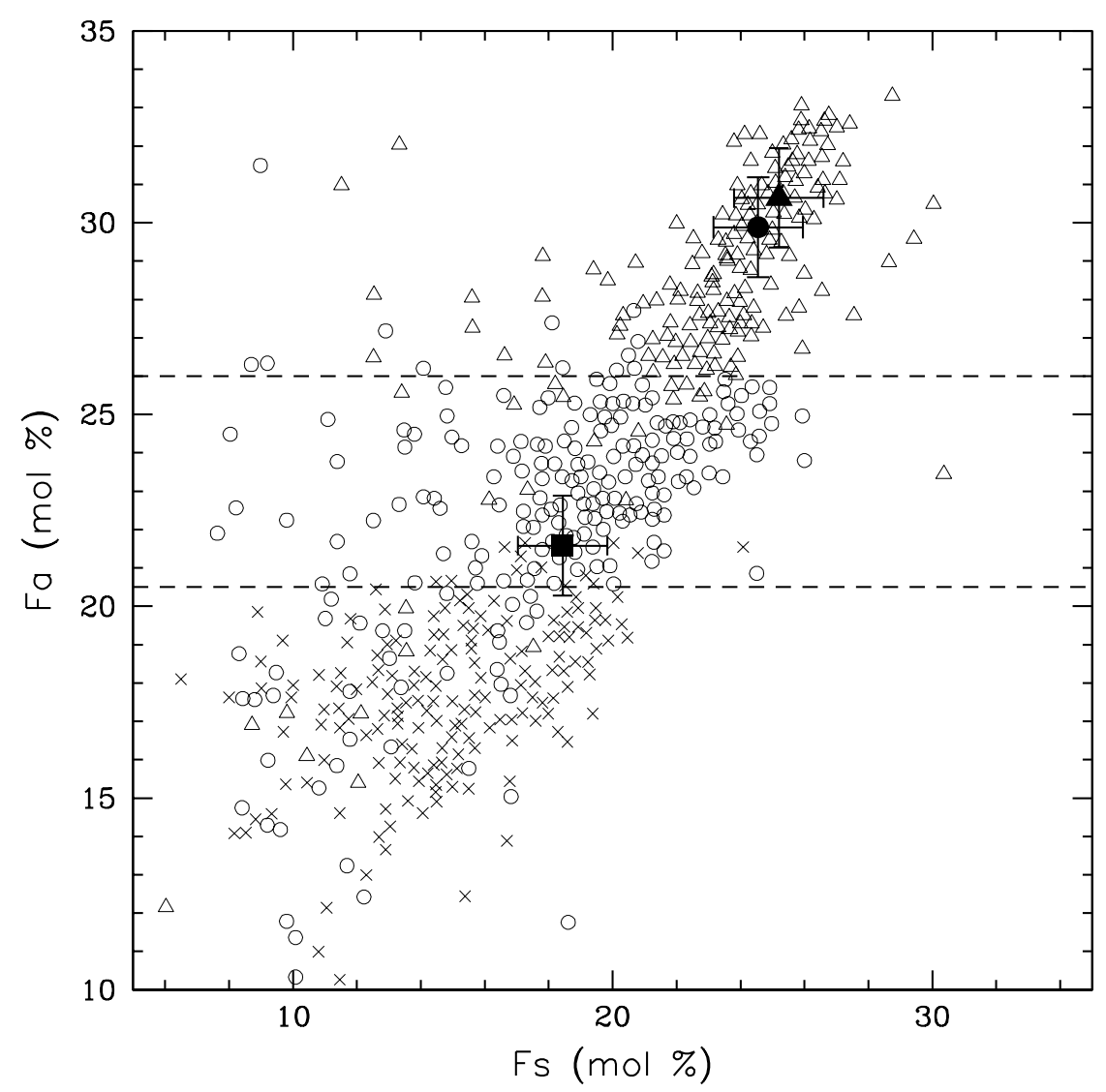

Figure 10: Molar contents of Fa vs. Fs for NEAs 241662 (2000 KO44) (filled triangle), 19764 (2000 NF5) (filled circle), 138404 (2000 HA24) (filled square), along with the values for LL (open triangles), $\mathrm{L}$ (open circles) and H (x) ordinary chondrites from Nakamura et al. (2011). The error bars correspond to the values determined by Dunn et al. (2010), $1.4 \mathrm{~mol} \%$ for Fs and $1.3 \mathrm{~mol} \%$ for Fa. The horizontal dashed-lines represent the approximate boundaries for LL, L, and $\mathrm{H}$ ordinary chondrites.

Table 3: Spectral band parameters for the asteroids studied. Molar contents of Fayalite (Fa), Ferrosilite $(\mathrm{Fs})$, wollastonite (Wo), and ol/(ol+px) ratio for each asteroid (where applicable) are presented.

\begin{tabular}{|c|c|c|c|c|c|c|c|}
\hline Object & $\begin{array}{c}\text { Band I center } \\
(\mu \mathrm{m})\end{array}$ & $\begin{array}{c}\text { Band II center } \\
(\mu \mathrm{m})\end{array}$ & BAR & $\begin{array}{c}\text { Fa } \\
(\mathrm{mol} \%)\end{array}$ & $\begin{array}{c}\text { Fs } \\
(\mathrm{mol} \%)\end{array}$ & $\begin{array}{c}\text { Wo } \\
(\mathrm{mol} \%)\end{array}$ & $\begin{array}{c}\mathrm{ol} /(\mathrm{ol}+\mathrm{px}) \\
\end{array}$ \\
\hline 5407 (1992 AX) & $0.93 \pm 0.01$ & $2.01 \pm 0.02$ & $2.31 \pm 0.06$ & - & $44.5 \pm 3.3 \%$ & $10.4 \pm 1.1 \%$ & - \\
4055 Magellan & $0.93 \pm 0.01$ & $1.92 \pm 0.02$ & $1.95 \pm 0.06$ & - & $34.9 \pm 3.3 \%$ & $6.7 \pm 1.1 \%$ & - \\
19764 (2000 NF5) & $1.01 \pm 0.01$ & $1.99 \pm 0.02$ & $0.26 \pm 0.06$ & $29.9 \pm 1.3 \%$ & $24.6 \pm 1.4 \%$ & - & $0.66 \pm 0.03$ \\
138404 (2000 HA24) & $0.95 \pm 0.01$ & $1.98 \pm 0.02$ & $0.84 \pm 0.06$ & $21.6 \pm 1.3 \%$ & $18.4 \pm 1.4 \%$ & - & $0.52 \pm 0.03$ \\
164121 (2003 YT1) & $0.93 \pm 0.01$ & $1.90 \pm 0.02$ & $1.64 \pm 0.06$ & - & $31.9 \pm 3.3 \%$ & $5.5 \pm 1.1 \%$ & - \\
241662 (2000 KO44) & $1.02 \pm 0.01$ & $1.99 \pm 0.02$ & $0.28 \pm 0.06$ & $30.7 \pm 1.3 \%$ & $25.2 \pm 1.4 \%$ & - & $0.66 \pm 0.03$ \\
\hline
\end{tabular}




\section{Conclusions}

We have found that all observed MC asteroids, five in total, belong to the S-complex, including the $\mathrm{S}, \mathrm{Sr}$ and $\mathrm{Sl}$ classes. The classifications assigned by us are consistent with previous work with the exception of two cases. This discrepancy could be attributed to atmospheric conditions or phase angle effects. Among the NEAs observed, seven belong to the S-complex, including the S, Sa, Sk and Sl classes. All of them have been classified for the first time in the present work. Two NEAs were classified as V-type asteroids.

The mineralogical analysis of NEAs 4055 Magellan and 164121 (2003 YT1), showed that their pyroxene chemistries are consistent with both, howardites and cumulate eucrites. Howardites are polymict breccia composed by a mixture of eucrite and diogenite fragments. Cumulate eucrites, on the other hand, are achondritic meteorites, which are thought to have formed in shallow magma chambers within the crust of their parent body, where they slowly crystallized. Howardites and eucrites along with diogenites (HED meteorites) have been linked to asteroid (4) Vesta, on the basis of their spectral and mineralogical similarities derived from groundbased observations (e.g., McCord et al., 1970; Consolmagno and Drake, 1977; Gaffey, 1997; Keil, 2002). This idea has been supported by recent spacecraft spectroscopic observations obtained by Dawn (e.g., Reddy et al., 2012b; De Sanctis et al., 2012), that shows Vesta as the most likely parent body of these meteorites. The Vesta asteroid family extends from the $\nu_{6}$ secular resonance to the 3:1 mean motion resonance with Jupiter, providing a viable route for the delivery of these objects to the near-Earth space (Wisdom, 1985; Bottke et al., 2002). Our results suggest that 4055 Magellan and 164121 (2003 YT1) are formed by regolith breccia originated in a larger asteroid that experienced extensive igneous processing. The pyroxene chemistries derived for these asteroids are similar to those found by Burbine et al. (2009) for a group of V-type NEAs. Although we cannot rule out a different parent body for these NEAs, these results are consistent with the aforementioned scenario, which would link Vesta with these asteroids via secular or mean motion resonances.

The mineralogical analysis of MC 5407 (1992 AX) revealed a pyroxene chemistry consistent with the range estimated for non-cumulate eucrites. These results suggest that the parent body of this asteroid experienced temperatures high enough to cause at least partial melting, making basaltic achondrites the most plausible meteorite analogs (Gaffey et al., 1993).

For NEAs, 241662 (2000 KO44), 19764 (2000 NF5) and 138404 (2000 HA24), the mineralogical analysis revealed ordinary chondrite-like compositions. In particular, 241662 (2000 KO44) and 19764 (2000 NF5) showed mineral compositions consistent with olivine-rich LL ordinary chondrites. Within the error bars the olivine and pyroxene chemistries of these two asteroids, as well as the olivine abundances, are consistent with the values measured from the returned samples of asteroid 25143 Itokawa. The mean compositions of olivine and low-Ca pyroxene for these samples gave values of $\mathrm{Fa}_{29}$ and $\mathrm{Fs}_{24}$, respectively (Nakamura et al., 2011), with an olivine abundance of $64 \%$ (Tsuchivama et al., 2011). The analysis of Itokawa samples also revealed that this asteroid is a breccia, consisting of poorly equilibrated LL4 and highly equilibrated LL5 and LL6 materials (Nakamura et al., 2011). This degree of thermal metamorphism indicates that the parent body where Itokawa originated must have had a diameter larger than $20 \mathrm{~km}$, necessary to reach temperatures of $\sim 800^{\circ} \mathrm{C}$ that can explain the range of petrologic types observed among the Itokawa particles (Nakamura et al., 2011). LL ordinary chondrites have been linked to the Flora family (Vernazza et al., 2008), which is located in the inner part of the main belt near the $\nu_{6}$ secular resonance. Although the derived silicate compositions of 241662 (2000 KO44) and 19764 (2000 NF5) are consistent with that of LL ordinary chondrites, we noticed that their olivine abundances $(66 \%)$ are lower than the ranges found by Vernazza et al. (2008) for LL chondrites (70-85\%), and members of the Flora family (76-82\%). However, this discrepancy could be explained by the fact that Vernazza et al. (2008) used a radiative transfer model 
(Shkuratov et al., 1999) to calculate the $o l /(o l+p x)$ ratios, while we used empirical spectral calibrations derived from the analysis of ordinary chondrites.

NEA 138404 (2000 HA24) is considered as a possible target for robotic and manned missions due to its low $\Delta \mathrm{V}$ (Landau and Strange, 2011). This asteroid showed a composition similar to $\mathrm{L}$ and $\mathrm{H}$ ordinary chondrites. If this NEA formed in a $\mathrm{L}$ ordinary chondrite parent body, then its origin could be related to the formation of the Gefion family. Nesvorný et al. (2009) found a possible link between this family (located at 2.7-2.82 AU) and L ordinary chondrites. Asteroids in the Gefion family are thought to have originated after an impact event that disrupted its parent body $\sim 470$ million years ago (Haack et al., 1996; Korochantseva et al., 2007). This group of objects exhibit spectral characteristics compatible with S-complex asteroids (Cellino et al., 2002). According to Nesvorný et al. (2009) the location of the Gefion family, near the 5:2 mean motion resonance with Jupiter, provides a viable source for the delivery of this material to Earth. Thus, objects in the Gefion family could eventually escape from the main belt and evolve to Earth-crossing orbits.

Another possible scenario for the origin of 138404 (2000 HA24) is that this object is a fragment of a $\mathrm{H}$ ordinary chondrite parent body. The main belt asteroid 6 Hebe, which is located at 2.426 AU between the $\nu_{6}$ secular resonance and the 3:1 mean motion resonance, shows spectral characteristics and mafic silicate compositions consistent with those of $\mathrm{H}$ chondrites (Gaffey, 1996; Gaffey and Gilbert, 1998), making of this asteroid its probably parent body. Cosmic ray exposure ages (CRE) of $\mathrm{H}$ chondrites indicate that their parent body, or a fragment of it, experienced multiple collisional events, occurred $\sim 60, \sim 33$ and $\sim 8$ million years ago (Graf and Marti, 1995). Numerical experiments have shown that fragments ejected from Hebe at velocities of a few hundreds $\mathrm{m} / \mathrm{s}$ can be transported into the resonance zones and from there into Earth-crossing orbits within a time span of $\sim 1$ million years (Farinella et al., 1993). Thus, a possible scenario for the origin of 138404 (2000 HA24) is that this object was ejected during one of the collisional events and later transported to the near-Earth space. If 138404 (2000 HA24) is indeed a fragment of Hebe, then it could be also linked to some of the $\mathrm{H}$ ordinary chondrites found on Earth. It is believed that the latest collisional event occurred $\sim 8$ million years ago is the source of $\sim 45 \%$ of $\mathrm{H}$ chondrites that reach the Earth (Graf and Marti, 1995). Since this collisional event took place within the typical lifetimes $\left(10^{6}-10^{7}\right.$ years $)$ associated with NEAs (Morbidelli et al., 2002), it is possible that some $\mathrm{H}$ chondrite meteorites were ejected from the surface of 138404 (2000 HA24).

The compositional information derived in this work from the analysis of VIS-NIR spectral data, along with dynamical considerations allowed us to discuss possible scenarios for the origin of the studied asteroids, as well as their connection with meteorites found on Earth. Since our sample included PHAs an a possible target for robotic and manned missions, the information gathered here could eventually help in planning future missions, and contribute in the development of mitigation strategies. 


\section{Acknowledgements}

This paper is based on data obtained with the New Technology Telescope at La Silla, Chile, observations collected at the Centro Astronómico Hispano Alemán (CAHA), operated jointly by the Max-Planck Institut für Astronomie and the Instituto de Astrofisica de Andalucia (CSIC), and observations acquired with the Infrared Telescope Facility on Mauna Kea, Hawai'i. Part of the data used in this work were obtained from the MIT-UH-IRTF Joint Campaign for NEO Reconnaissance. The authors would like to thank Olivier Hainaut for his help during the observations at the NTT. JAS research was supported by a PhD fellowship of the International Max Planck Research School on Physical Processes in the Solar System and Beyond, and the Deutsche Forschungsgemeinschaft. VR research was supported by NASA 182 NEOO Program Grant NNX12AG12G, and NASA Planetary Geology and Geophysics Grant NNX11AN84G. We thank the IRTF TAC for awarding time to this project, and to the IRTF TOs and MKSS staff for their support. The authors would like to thank Paul Abell and an anonymous reviewer for their reviews, which helped to improve the manuscript.

\section{References}

Abell, P.A., Gaffey, M.J., Hardersen, P.S., 2004. Compositional Results of Binary Near-Earth Asteroid 2003 YT1: A Basaltic Achondrite, in: AAS/Division for Planetary Sciences Meeting Abstracts \#36, p. 1132.

Abell, P.A., Korsmeyer, D.J., Landis, R.R., Jones, T.D., Adamo, D.R., Morrison, D.D., Lemke, L.G., Gonzales, A.A., Gershman, R., Sweetser, T.H., Johnson, L.L., Lu, E., 2009. Scientific exploration of near-Earth objects via the Orion Crew Exploration Vehicle. Meteoritics and Planetary Science 44, 1825-1836.

Adams, J.B., 1974. Visible and near-infrared diffuse reflectance spectra of pyroxenes as applied to remote sensing of solid objects in the solar system. Journal of Geophysical Research 79, $4829-4836$.

Adams, J.B., 1975. Interpretation of visible and near-infrared diffuse reflectance spectra of pyroxenes and other rock-forming minerals, in: Karr, C., I. (Ed.), Infrared and Raman Spectroscopy of Lunar and Terrestrial Minerals. Academic, New York, pp. 91-116.

Angeli, C.A., Lazzaro, D., 2002. Spectral properties of Mars-crossers and near-Earth objects. Results of the $\mathrm{S}^{3} \mathrm{OS}^{2}$ survey. Astronomy and Astrophysics 391, 757-765.

Binzel, R.P., Lupishko, D., di Martino, M., Whiteley, R.J., Hahn, G.J., 2002. Physical Properties of Near-Earth Objects. Asteroids III , 255-271.

Binzel, R.P., Rivkin, A.S., Stuart, J.S., Harris, A.W., Bus, S.J., Burbine, T.H., 2004. Observed spectral properties of near-Earth objects: results for population distribution, source regions, and space weathering processes. Icarus 170, 259-294.

Bottke, W.F., Jedicke, R., Morbidelli, A., Petit, J.M., Gladman, B., 2000. Understanding the Distribution of Near-Earth Asteroids. Science 288, 2190-2194.

Bottke, Jr., W.F., Vokrouhlický, D., Rubincam, D.P., Broz, M., 2002. The Effect of Yarkovsky Thermal Forces on the Dynamical Evolution of Asteroids and Meteoroids. Asteroids III , 395-408. 
Burbine, T.H., Buchanan, P.C., Binzel, R.P., 2007. Deriving Formulas from HED Spectra for Determining the Pyroxene Mineralogy of Vesta and Vestoids, in: Lunar and Planetary Institute Science Conference Abstracts, p. 2117.

Burbine, T.H., Buchanan, P.C., Dolkar, T., Binzel, R.P., 2009. Pyroxene mineralogies of nearEarth vestoids. Meteoritics and Planetary Science 44, 1331-1341.

Burbine, T.H., McCoy, T.J., Meibom, A., Gladman, B., Keil, K., 2002. Meteoritic Parent Bodies: Their Number and Identification, in: Bottke, W., Cellino, A., Paolicchi, P., Binzel, R. (Eds.), Asteroids III. University of Arizona Press, pp. 653-667.

Burns, R.G., 1993. Mineralogical Applications of Crystal Field Theory. Cambridge, UK: Cambridge University Press, 575pp.

Bus, S.J., 1999. Compositional structure in the asteroid belt: Results of a spectroscopic survey. Ph.D. thesis. Massachusetts Institute of Technology.

Bus, S.J., Binzel, R.P., 2002a. Phase II of the Small Main-Belt Asteroid Spectroscopic Survey A Feature-Based Taxonomy. Icarus 158, 146-177.

Bus, S.J., Binzel, R.P., 2002b. Phase II of the Small Main-Belt Asteroid Spectroscopic Survey The Observations. Icarus 158, 106-145.

Bus, S.J., Vilas, F., Barucci, M.A., 2002. Visible-Wavelength Spectroscopy of Asteroids, in: Bottke, W., Cellino, A., Paolicchi, P., Binzel, R. (Eds.), Asteroids III. University of Arizona Press, pp. 169-182.

Cellino, A., Bus, S.J., Doressoundiram, A., Lazzaro, D., 2002. Spectroscopic Properties of Asteroid Families, in: Bottke, W., Cellino, A., Paolicchi, P., Binzel, R. (Eds.), Asteroids III. University of Arizona Press, pp. 633-643.

Chapman, C.R., Morrison, D., 1994. Impacts on the Earth by asteroids and comets: assessing the hazard. Nature 367, 33-40.

Cloutis, E.A., Gaffey, M.J., 1991. Pyroxene spectroscopy revisited - Spectral-compositional correlations and relationship to geothermometry. Journal of Geophysical Research 96, 22809.

Cloutis, E.A., Gaffey, M.J., Jackowski, T.L., Reed, K.L., 1986. Calibrations of phase abundance, composition, and particle size distribution for olivine-orthopyroxene mixtures from reflectance spectra. Journal of Geophysical Research 91, 11641-11653.

Consolmagno, G.J., Drake, M.J., 1977. Composition and evolution of the eucrite parent body - Evidence from rare earth elements. Geochimica et Cosmochimica Acta 41, 1271-1282.

Cushing, M.C., Vacca, W.D., Rayner, J.T., 2004. Spextool: A Spectral Extraction Package for SpeX, a 0.8-5.5 Micron Cross-Dispersed Spectrograph. Publications of the Astronomical Society of the Pacific 116, 362-376.

de León, J., Licandro, J., Serra-Ricart, M., Pinilla-Alonso, N., Campins, H., 2010. Observations, compositional, and physical characterization of near-Earth and Mars-crosser asteroids from a spectroscopic survey. Astronomy and Astrophysics 517, A23. 
De Sanctis, M.C., Ammannito, E., Capria, M.T., Tosi, F., Capaccioni, F., Zambon, F., Carraro, F., Fonte, S., Frigeri, A., Jaumann, R., Magni, G., Marchi, S., McCord, T.B., McFadden, L.A., McSween, H.Y., Mittlefehldt, D.W., Nathues, A., Palomba, E., Pieters, C.M., Raymond, C.A., Russell, C.T., Toplis, M.J., Turrini, D., 2012. Spectroscopic Characterization of Mineralogy and Its Diversity Across Vesta. Science 336, 697-700.

Dunn, T.L., Burbine, T.H., Bottke, W.F., Clark, J.P., 2013. Mineralogies and source regions of near-Earth asteroids. Icarus 222, 273-282.

Dunn, T.L., McCoy, T.J., Sunshine, J.M., McSween, H.Y., 2010. A coordinated spectral, mineralogical, and compositional study of ordinary chondrites. Icarus 208, 789-797.

Farinella, P., Froeschle, C., Gonczi, R., 1993. Meteorites from the asteroid 6 Hebe. Celestial Mechanics and Dynamical Astronomy 56, 287-305.

Gaffey, M.J., 1996. Spectral Identification of Asteroid 6 Hebe as the Mainbelt Parent Body of the H-type Ordinary Chondrites. Meteoritics and Planetary Science Supplement 31, 47.

Gaffey, M.J., 1997. Surface Lithologic Heterogeneity of Asteroid 4 Vesta. Icarus 127, 130-157.

Gaffey, M.J., Burbine, T.H., Piatek, J.L., Reed, K.L., Chaky, D.A., Bell, J.F., Brown, R.H., 1993. Mineralogical variations within the S-type asteroid class. Icarus 106, 573-602.

Gaffey, M.J., Cloutis, E.A., Kelley, M.S., Reed, K.L., 2002. Mineralogy of Asteroids, in: Bottke, W., Cellino, A., Paolicchi, P., Binzel, R. (Eds.), Asteroids III. University of Arizona Press, pp. 183-204.

Gaffey, M.J., Gilbert, S.L., 1998. Asteroid 6 Hebe: The probable parent body of the H-Type ordinary chondrites and the IIE iron meteorites. Meteoritics and Planetary Science 33, 12811295 .

Graf, T., Marti, K., 1995. Collisional history of H chondrites. Journal of Geophysical Research 100, 21247-21264.

Haack, H., Farinella, P., Scott, E.R.D., Keil, K., 1996. Meteoritic, Asteroidal, and Theoretical Constraints on the 500 MA Disruption of the L Chondrite Parent Body. Icarus 119, 182-191.

Keil, K., 2002. Geological History of Asteroid 4 Vesta: The "Smallest Terrestrial Planet", in: Bottke, W., Cellino, A., Paolicchi, P., Binzel, R. (Eds.), Asteroids III. University of Arizona Press, pp. 573-584.

Korochantseva, E.V., Trieloff, M., Lorenz, C.A., Buykin, A.I., Ivanova, M.A., Schwarz, W.H., Hopp, J., Jessberger, E.K., 2007. L-chondrite asteroid breakup tied to Ordovician meteorite shower by multiple isochron 40Ar-39Ar dating. Meteoritics and Planetary Science 42, 113130.

Landau, D., Strange, N., 2011. Near-Earth Asteroids Accessible to Human Exploration With High-Power Electric Propulsion. AAS , 11-46.

Lazzaro, D., Angeli, C.A., Carvano, J.M., Mothé-Diniz, T., Duffard, R., Florczak, M., 2004. $\mathrm{S}^{3} \mathrm{OS}^{2}$ : the visible spectroscopic survey of 820 asteroids. Icarus 172, 179-220.

McCord, T.B., Adams, J.B., Johnson, T.V., 1970. Asteroid Vesta: Spectral Reflectivity and Compositional Implications. Science 168, 1445-1447. 
Michel, P., Migliorini, F., Morbidelli, A., Zappalà, V., 2000. The population of Mars-Crossers: Classification and dynamical evolution. Icarus 145, 332-347.

Michelsen, R., Nathues, A., Lagerkvist, C.I., 2006. Spectroscopy of near-Earth asteroids. Astronomy and Astrophysics 451, 331-337.

Mittlefehldt, D.W., McCoy, T.J., Goodrich, C.A., Kracher, A., 1998. Non-Chondritic Meteorites from Asteroidal Bodies, in: Papike, J. (Ed.), Reviews in Mineralogy Vol. 36, Planetary Materials. Mineralogical Society of America, pp. 4-001-4-196.

Morbidelli, A., Bottke, Jr., W.F., Froeschlé, C., Michel, P., 2002. Origin and Evolution of Near-Earth Objects. Asteroids III , 409-422.

Morbidelli, A., Nesvorny, D., 1999. Numerous Weak Resonances Drive Asteroids toward Terrestrial Planets Orbits. Icarus 139, 295-308.

Morrison, D., Harris, A.W., Sommer, G., Chapman, C.R., Carusi, A., 2002. Dealing with the Impact Hazard. Asteroids III , 739-754.

Moskovitz, N.A., Willman, M., Burbine, T.H., Binzel, R.P., Bus, S.J., 2010. A spectroscopic comparison of HED meteorites and V-type asteroids in the inner Main Belt. Icarus 208, $773-788$.

Nakamura, T., Noguchi, T., Tanaka, M., Zolensky, M.E., Kimura, M., Tsuchiyama, A., Nakato, A., Ogami, T., Ishida, H., Uesugi, M., Yada, T., Shirai, K., Fujimura, A., Okazaki, R., Sandford, S.A., Ishibashi, Y., Abe, M., Okada, T., Ueno, M., Mukai, T., Yoshikawa, M., Kawaguchi, J., 2011. Itokawa Dust Particles: A Direct Link Between S-Type Asteroids and Ordinary Chondrites. Science 333, 1113-1116.

Nathues, A., 2010. Spectral study of the Eunomia asteroid family Part II: The small bodies. Icarus 208, 252-275.

Nesvorný, D., Vokrouhlický, D., Morbidelli, A., Bottke, W.F., 2009. Asteroidal source of L chondrite meteorites. Icarus 200, 698-701.

Rayner, J.T., Toomey, D.W., Onaka, P.M., Denault, A.J., Stahlberger, W.E., Vacca, W.D., Cushing, M.C., Wang, S., 2003. SpeX: A Medium-Resolution 0.8-5.5 Micron Spectrograph and Imager for the NASA Infrared Telescope Facility. Publications of the Astronomical Society of the Pacific 115, 362-382.

Reddy, V., Corre, L.L., Hicks, M., Lawrence, K., Buratti, B.J., Abell, P.A., Gaffey, M.J., Hardersen, P.S., 2012a. Composition of near-Earth Asteroid 2008 EV5: Potential target for robotic and human exploration. Icarus 221, 678-681.

Reddy, V., Nathues, A., Le Corre, L., Sierks, H., Li, J.Y., Gaskell, R., McCoy, T., Beck, A.W., Schröder, S.E., Pieters, C.M., Becker, K.J., Buratti, B.J., Denevi, B., Blewett, D.T., Christensen, U., Gaffey, M.J., Gutierrez-Marques, P., Hicks, M., Keller, H.U., Maue, T., Mottola, S., McFadden, L.A., McSween, H.Y., Mittlefehldt, D., O’Brien, D.P., Raymond, C., Russell, C., 2012b. Color and Albedo Heterogeneity of Vesta from Dawn. Science 336, 700-704.

Reddy, V., Sanchez, J.A., Nathues, A., Moskovitz, N.A., Li, J.Y., Cloutis, E.A., Archer, K., Tucker, R.A., Gaffey, M.J., Paul Mann, J., Sierks, H., Schade, U., 2012c. Photometric, spectral phase and temperature effects on 4 Vesta and HED meteorites: Implications for the Dawn mission. Icarus 217, 153-168. 
Sanchez, J.A., Reddy, V., Nathues, A., Cloutis, E.A., Mann, P., Hiesinger, H., 2012. Phase reddening on near-Earth asteroids: Implications for mineralogical analysis, space weathering and taxonomic classification. Icarus 220, 36-50.

Sears, D.W.G., Brownlee, D.D., Pieters, C., Lindstrom, M., Britt, D., Clark, B.C., Gefert, L., Gorevan, S., Preble, J.C., 2000. A Multiple Near-Earth Asteroid Sample Return Mission Called Hera. Meteoritics and Planetary Science Supplement 35, 145.

Shkuratov, Y., Starukhina, L., Hoffmann, H., Arnold, G., 1999. A Model of Spectral Albedo of Particulate Surfaces: Implications for Optical Properties of the Moon. Icarus 137, 235-246.

Shoemaker, E.M., Williams, J.G., Helin, E.F., Wolfe, R.F., 1979. Earth-crossing asteroids - Orbital classes, collision rates with earth, and origin, in: Gehrels, T. (Ed.), Asteroids. University of Arizona Press, pp. 253-282.

Tsuchiyama, A., Uesugi, M., Matsushima, T., Michikami, T., Kadono, T., Nakamura, T., Uesugi, K., Nakano, T., Sandford, S.A., Noguchi, R., Matsumoto, T., Matsuno, J., Nagano, T., Imai, Y., Takeuchi, A., Suzuki, Y., Ogami, T., Katagiri, J., Ebihara, M., Ireland, T.R., Kitajima, F., Nagao, K., Naraoka, H., Noguchi, T., Okazaki, R., Yurimoto, H., Zolensky, M.E., Mukai, T., Abe, M., Yada, T., Fujimura, A., Yoshikawa, M., Kawaguchi, J., 2011. Three-Dimensional Structure of Hayabusa Samples: Origin and Evolution of Itokawa Regolith. Science 333, 1125-1128.

Tüg, H., 1977. Vertical Extinction on La Silla. The Messenger 11, 7-8.

Vernazza, P., Binzel, R.P., Thomas, C.A., DeMeo, F.E., Bus, S.J., Rivkin, A.S., Tokunaga, A.T., 2008. Compositional differences between meteorites and near-Earth asteroids. Nature $454,858-860$.

Wisdom, J., 1985. Meteorites may follow a chaotic route to earth. Nature 315, 731-733. 